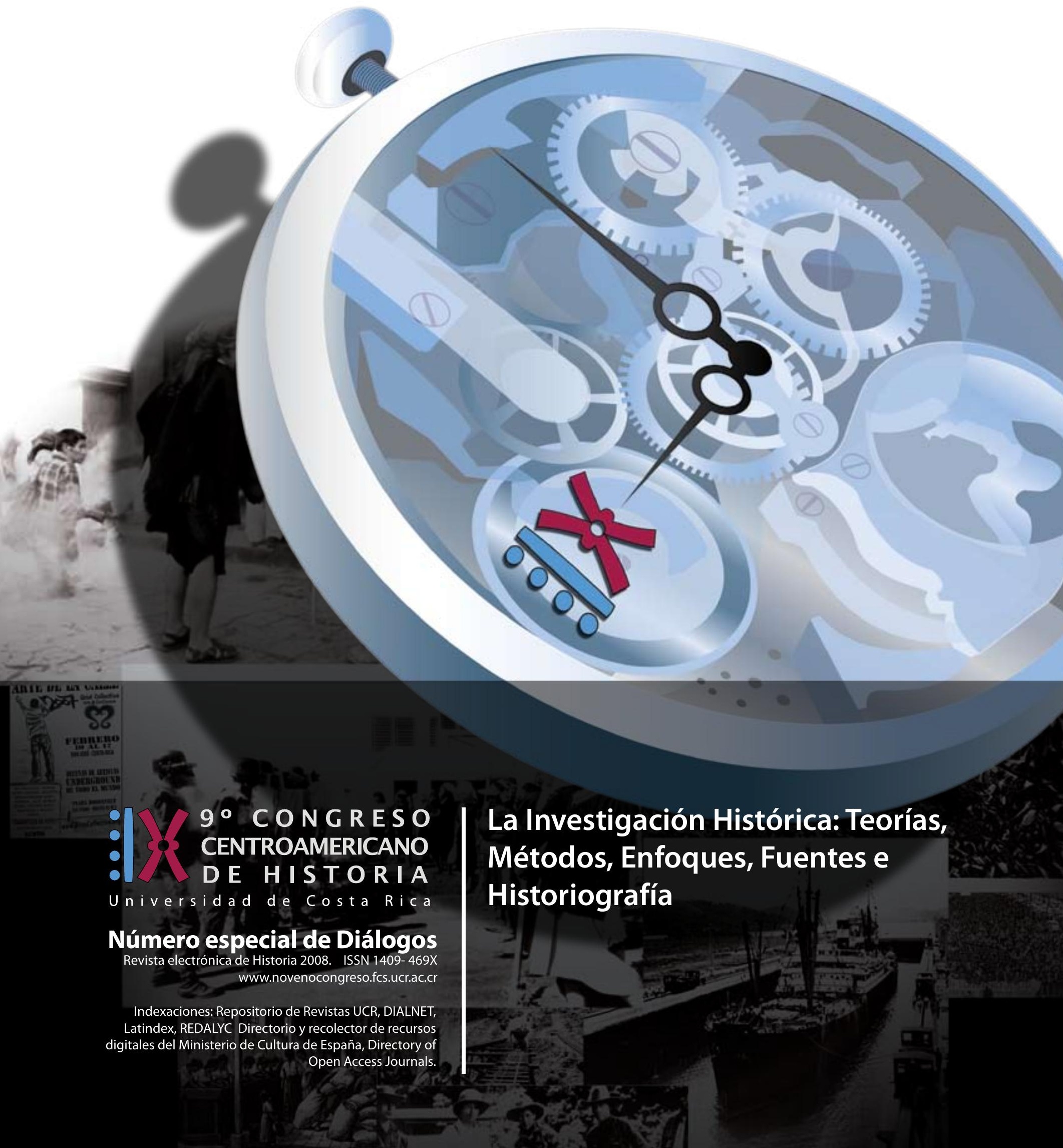


Indexaciones: Repositorio de Revistas UCR, DIALNET, Latindex, REDALYC Directorio y recolector de recursos digitales del Ministerio de Cultura de España, Directory of Open Access Journals. Diálogos Revista Electrónica de Historia ISSN 1409-469X. Número especial 2008. Dirección web: http://historia.fcs.ucr.ac.cr/dialogos.htm

\title{
El uso del Método Galarziano en dos documentos pictográficos del centro de México
}

\author{
Joel Ortiz Arroyo
}

LICENCIADO EN ETNOHISTORIA EN LA
ESCUELA NACIONAL DE ANTROPOLOGÍA E
HISTORIA
INVESTIGADOR EN EL CENTRO DE

INVESTIGACIONES Y ESTUDIOS SUPERIORES EN

ANTROPOLOGÍA SOCIAL

yumkaax4@hotmail.com 


\section{INTRODUCCIÓN}

El presente trabajo abordará el tema de la escritura tradicional nahua en dos códices de contenido económico elaborados en el centro de México durante el siglo XVI, en los que fue registrado el pago de tributos de forma local, así como los personajes que se ocupaban de la recaudación y el personaje a quien era entregado el total del tributo.

Para la realización del mismo estudio nos apoyamos en fuentes históricas como los cronistas del siglo XVI y en investigaciones recientes que tratan sobre el sistema de tributo y la importancia que tuvo este en la época prehispánica y posteriormente en época de la colonia; lo cual ayudó en la realización de la lectura de los códices.

La lectura de estas dos pictografías se realizó bajo el método propuesto por el Dr. Joaquín Galarza, el cual está basado en la lógica del pensamiento indígena nahua, que es el origen de los códices. Este método tiene como sustento el análisis sistemático y exhaustivo de la totalidad de los elementos pintados en los códices y está creado de tal manera que puede ser aplicable a los diferentes grupos de manuscritos indígenas tradicionales. 
Si bien ya se comentó que se trabajará con códices que fueron elaborados en el centro de México, es entonces necesario, hacer una somera descripción de lo que son estos documentos, para lo cual se tomará en cuenta la definición de uno de los principales investigadores del tema, el Doctor Joaquín Galarza, quien nos refiere que los códices son:

"los manuscritos de los indígenas mesoamericanos que fijaron sus lenguas por medio de un sistema básico del empleo de la imagen codificada" ${ }^{1}$

Este sistema de escritura tuvo como propósito el registro de diferentes temas, se sabe que los documentos pictográficos son de una variedad inmensa y que algunos de estos pueden tratar sobre una o más materias a la vez. De esta forma se pueden catalogar códices de contenido histórico, religioso, geográfico, económico, entre otros muchos más temas.

El soporte en el que fueron elaborados los códices también es diverso, para la época prehispánica se conoce que fueron utilizadas diferentes fibras, como la del algodón, amate y maguey, aunque también fueron empleadas pieles de animales como la del venado. Durante la época de la colonia estos materiales fueron reemplazados por papel europeo y telas industriales.

Acerca de los colores con los que se pintaban estos códices, es sabido que fueron extraídos básicamente de vegetales, minerales y animales, los cuales también fueron utilizados en la primera etapa colonial.

Los códices que fueron elaborados durante el siglo XVI presentan un rasgo característico, la combinación de dos sistemas de escritura. Por una parte se encuentra el sistema pictográfico

1 Joaquín Galarza, Amatl, Amoxtli, el papel, el libro. Los códices mesoamericanos. Guía para la introducción al estudio del material pictórico indígena (México: Editorial Tava, 2ª edición, 1990), 15. 
tradicional nahua y por otro el de caracteres latinos en náhuatl y español, a estos documentos se les ha denominado como códices mixtos, como ejemplo de esta categoría de documentos, tenemos los códices 028 y 108 .

La función social de los códices era la de registrar y dar a conocer información sobre diferentes temas, en especial sobre hechos históricos, religiosos, sociales, sobre asuntos geográficos, calendáricos o sucesos importantes de la época como la entronización de gobernantes, guerras y eclipses. Sobre los códices y la escritura se ejerció cierto control por parte de la nobleza, pues esta intentaba legitimar y afianzarse al poder a través de la historia escrita en estos documentos. Siguiendo el orden de esta idea, C. Arellano, inscribe que posiblemente los tlacuilos o escribas, quienes se encargaron de pintar los códices, hayan pertenecido al estrato de la nobleza, puesto que dicho estrato fue el único instruido en la lectura y escritura, aunque termina resaltando que los hijos de los nobles no eran los únicos que podían accesar al conocimiento de la escritura, pues el ingreso a la escuela no estaba determinado por condiciones sociales o económicas, sino por las aptitudes de los niños. ${ }^{2}$

Como se escribió con antelación, la presente ponencia trata sobre el análisis de dos códices, catalogados por la Biblioteca Nacional de Francia con los números 028 y 108, estos códices son conocidos respectivamente como CONTRIBUCIONES O TRIBUTOS DE TLAXINCAN, TLAYLOTLACAN, TECPAN, ETC y TRIBUTOS DE TLAXINICAN, TLAYLOTLACAN, TECPANPA, ETC., los cuales presentan en su contenido una temática económica, pues en ellos se registró una lista de tributos que contiene productos como pescados, canoas, guajolotes, gallinas

2 Carmen Arellano Hoffmann, $\square$ El escriba mesoamericano y sus utensilios de trabajo. La posición social del escriba antes y después de la conquista española $\square$ en Libros y escritura de tradición indígena, Ensayos sobre códices prehispánicos y coloniales de México, ed. Peer Schmidt y Xavier Noguéz (México, 2002) 219, 220. 
de castilla, vasijas, pulque (que es un licor fermentado del maguey) y monedas, los cuales fueron pagados por los habitantes de diez barrios a un personaje de nombre Joseph Jorgen.

La descripción física de los códices que dan algunos investigadores es la siguiente.

El códice 028 tiene formato de tira, el soporte en el que fue hecho es de papel amate, el color de la pintura utilizada en este documento fue el negro, dicho documento mide cuarenta centímetros de largo por 38 centímetros de ancho. Por su parte el documento 108 tiene un formato de tira de ciento cuarenta centímetros de largo por treinta y uno de ancho, en cuanto a su soporte, se menciona que fue hecho sobre papel europeo, el color de tinta utilizado en este códice fue el sepia.

Siendo el centro de México el lugar donde se elaboraron estos códices, es importante resaltar el lugar geográfico de donde provienen los mismos. Al centro del Altiplano de México se formó una cuenca, la cual se componía por varios ejes volcánicos, según la descripción de la misma, hecha por M. Serra Puche, "la Cuenca de México se convierte en una planicie elevada rodeada al este por la Sierra Nevada, al oeste por la Sierra de las Cruces y al sur por la Sierra del Ajusco el lado norte tiene una serie de colinas bajas y discontinuas" ${ }^{3}$

A razón de G. Espinosa, la zona lacustre posiblemente se formó por el cauce de dos ríos que desembocaban en el río balsas, en la zona occidental de México. La formación del lago de la cuenca, comenta, fue parcial, puesto que no todas las zonas de la cuenca se cerraron al mismo tiempo, lo cual implicaba que la profundidad variara de una zona a otra. ${ }^{4}$

3 Mari Carmen Serra Puche, Los recursos lacustres de la cuenca de México durante el formativo (México: Coordinación general de estudios de posgrado, Instituto de Investigaciones Antropológicas, colección de posgrado 3, $1^{\text {a }}$ edición, UNAM, 1988), 21.

4 Gabriel Espinosa Pineda, El embrujo del lago. El sistema lacustre de la cuenca de México en 
Indexaciones: Repositorio de Revistas UCR, DIALNET, Latindex, REDALYC Directorio y recolector de recursos digitales del Ministerio de Cultura de España, Directory of Open Access Journals.

Diálogos Revista Electrónica de Historia ISSN 1409-469X. Número especial 2008. Dirección web: http://historia.fcs.ucr.ac.cr/dialogos.htm

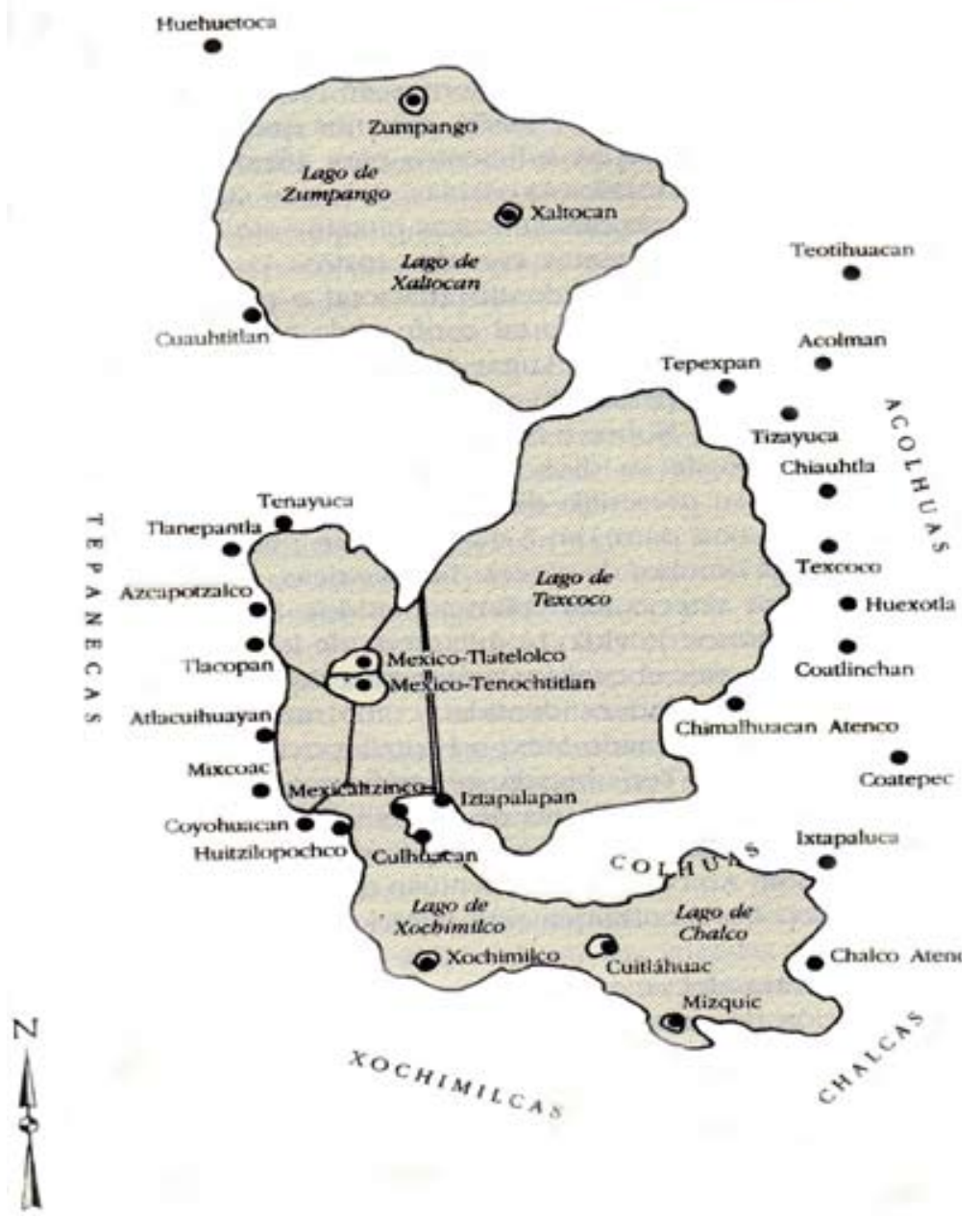

Imagen 1. Mapa La Cuenca de México

de Alfredo López Austin y Leonardo López Luján

La somera descripción que se ha dado sobre la cuenca, sirve para ubicar dentro de la misma

la cosmovisión mexica (México: Instituto de Investigaciones Históricas e Instituto de Investigaciones Antropológicas, $1^{\text {a }}$ edición, UNAM, 1996), 26. 
a los diez "barrios" que fueron pintados en ambos códices, cada uno de ellos aparece con su topónimo, que es el glifo que da nombre al sitio en referencia, y nombre escrito con caracteres latinos. Se toma en cuenta el concepto de "barrio" porque es así como aparece señalado en una de las glosas del códice número 108. Sin embargo, resulta importante aclarar lo que se entiende por el concepto de barrio.

De acuerdo con lo expresado por P. Carrasco y con lo cual estamos de acuerdo, el barrio o calpulli, es una unidad corporativa con administración comunal de la tierra y responsabilidad colectiva en el pago de tributos. El barrio aunque es una unidad comunal, se sabe que fue administrada por la élite y no por la comunidad basada en el parentesco como se planteó por mucho tiempo. ${ }^{5}$

Ya que hemos mencionado que el tributo pintado en los códices 028 y 108 fue pagado por los habitantes de diez barrios, nos parece importante ubicarlos geográficamente, para ello se comentó acerca de lo que fue la cuenca de México en la época prehispánica; valiéndonos de investigaciones sobre la toponímia, geografía, diccionarios de lengua nahuatl y crónicas del siglo XVI pudimos aclarar la ubicación de algunos de los diez barrios antes mencionados.

Cabe hacer la aclaración de que los siete barrios que aparecen en el códice 028 son parte también del códice 108; esta segunda pictografía se complementa con tres topónimos más, dando el total de los 10 barrios ya mencionados.

Tlaxincan es el primero de los "barrios", fue representado con el topónimo de una pequeña hacha para cortar madera. E. Boban, traduce este topónimo como "taller de carpinteros"6; R.

5 Pedro Carrasco, "La economía del México prehispánico" en Economía política e ideología en el México prehispánico, editores Pedro Carrasco y Johanna Broda (México: Centro de Investigaciones Superiores del INAH, SEP, $1^{\text {a }}$ edición, 1978), 39.

6 Eugene Boban, "Piéces judiciaires ordonnances royales, etc. $\mathrm{N}^{\circ} 108$, Contributions ou 
Siméon, ${ }^{7}$ da la misma acepción pero ninguno de los dos da la ubicación de la localidad. En el documento catalogado con el número 107, conocido con el nombre PLANO TOPOGRÁFICO DE TEXCOCO, se hace referencia a una localidad con el nombre de Tlaxincan, lo que hace pensar que posiblemente se trate del mismo barrio, por lo tanto se puede situar a este topónimo dentro de la región de Texcoco.

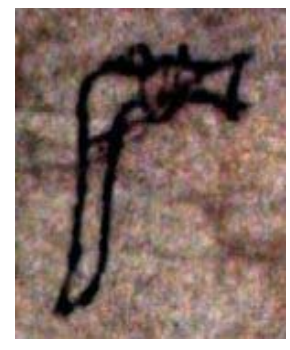

Imagen 2. Topónimo de Tlaxincan, Códice 028, BNF.

El segundo "barrio" es el de Tlaylotlacan, aparece registrado a través de una concha vista de frente. E. Boban, dice que Tlaylotlacan o más bien Tlailotlacan, es uno de los antiguos barrios de la villa de Texcoco ${ }^{8}$; R. Siméon, traduce el nombre como "los que regresan" y señala que es un barrio de la misma ciudad de Texcoco que recibió el nombre de los tlailotlaque ${ }^{9}$; P. Gerhard, nos dice que dentro de la ciudad de Texcoco existía un pueblo con el nombre de Santa María Tlailotlacan, el cual había alcanzado la categoría de cabecera ${ }^{10}$; F. Hicks, menciona que en tiempos de Nezahualcoyotl la ciudad de Texcoco fue dividida en seis parcialidades, una de ellas

tributs", en Catálogo de Boban, Documents pour server al histoire du mexique, (Francia: libro segundo, volumen segundo, 1891), 287.

7 Siméon Rémi, Diccionario de la lengua náhuatl o mexicana (editorial Siglo Veintiuno, $1^{\text {a }}$ edición en español, 1997), 698.

8 Boban, 288.

9 Siméon, 589.

10 Peter Gerhard, Geografia histórica de la Nueva España 1519-1821 (México: UNAM, 1986), 323. 
fue Tlailotlacan, nombre derivado del grupo étnico que arribó a Acolhuacan en el siglo $\mathrm{XV}^{11}$; P. Carrasco, dice que este barrio étnico se encontraba localizado en la zona central y que para fines del siglo XVII todavía sobrevivían barrios y ermitas con nombres que denotaban su origen ${ }^{12}$. Con lo antes mencionado se puede ubicar entonces a Tlaylotlacan como un barrio dentro de la zona de Texcoco.

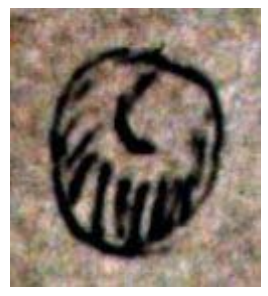

Imagen 3. Topónimo de Tlaylotlacan, Códice 028, BNF.

El tercer topónimo es Tecpanpa, representado por medio de un recinto visto de perfil, del cual puede verse el dintel y la jamba. E. Boban, señala que el nombre de Tecpanpa deriva de tecpan, "morada real o palacio" y de la preposición $p a$, que significa "hacia en o de"13; R. Siméon, citando a Clavijero, anota que Tecpan es uno de los barrios de Tenochtitlan que comprendía la parte sureste de la ciudad ${ }^{14}$; mientras que $\mathrm{CH}$. Gibson, nos dice que Xochimilco estaba integrado por tres gobiernos dinásticos con tres tlatoque o gobernantes, una de las subdivisiones era Tecpan, aunque también esta misma localidad era reconocida como barrio ${ }^{15}$; por su parte, D.

11 Frederic Hicks, "Los calpixque de Nezahualcoyotl" en Estudios de cultura nahuatl, vol. 14 (México: Instituto de Investigaciones Históricas, UNAM, 1980), 150.

12 Pedro Carrasco, "La Triple Alianza. Organización política y estructura territorial" en Temas Mesoamericanos, coordinadores Sonia Lombardo y Enrique Nalda (México: INAH, CNCA, $1^{\mathrm{a}}$ edición, 1996), 176.

13 Boban, 289.

14 Siméon, 450. 
Chimalpáhin, también reconoce como parte de Xochimilco a la localidad de Tecpan, puesto que menciona que Xochimilco se dividía en tres parcialidades siendo Tecpan una de ellas ${ }^{16}$.

Con la información citada por los anteriores autores, no se puede saber con exactitud la ubicación geográfica de este topónimo, pues lo ubican físicamente en dos lugares distintos, Tenochtitlan y Xochimilco; recordemos que también existe un lugar con el mismo nombre en Tlatelolco. Dentro de la MATRÍCULA DE TRIBUTOS, ${ }^{17}$ específicamente en la lámina número 12 , se encuentra pintado el topónimo de Tecpan; J. Corona, señala que esta lámina contiene glifos de pueblos que formaban la provincia de Quahuacan, los cuales, en época prehispánica estaban bajo el dominio tepaneca ${ }^{18}$; según el mismo autor, estos pueblos están comprendidos en el actual Estado de México; mientras que M. Sepúlveda, ubica los topónimos de esta lámina dentro del actual Distrito Federal y Estado de México ${ }^{19}$.

Por los datos tan ambiguos que tenemos, no podemos inclinarnos a ubicar en alguna de estas dos grandes ciudades (Tenochtitlan y Xochimilco) al topónimo de Tecpan. Aunque por el tipo de tributo que se registra en los códices y que corresponde a lo que tenía que pagar este barrio, y por la posición de ambas ciudades antes señaladas, que se encuentran dentro del lago de la Cuenca de México, podemos dejar abierta la posible ubicación del topónimo dentro de cualquiera de las dos ciudades.

15 Charles Gibson, Los aztecas bajo el dominio español 1519-1810 (México: UNAM, 1980), 45.

16 Domingo, Chimalpáhin, Las ocho relaciones y el memorial de Colhuacan (México: Cien de México, CNCA, 1998), 291.

17 Es un códice de tributo a nivel estatal que muestra en su contenido a los pueblos que fueron las provincias que debían pagar tributo, además del tipo de tributo que pagaban.

18 Códice Matrícula de Tributos, palabras preliminares, Antonio Ortiz Mena, interpretación y notas José Corona Núñez (México: SHCP, $1^{\text {a }}$ edición, 1968), 32.

19 María Teresa Sepúlveda y Herrera, "La Matrícula de Tributos", Revista Arqueología Mexicana, Edición Especial, Serie Códices (México) (2003): 44. 


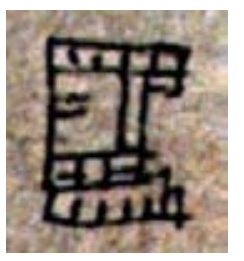

Imagen 4. Topónimo de Tecpanpa, Códice 028, BNF.

Tenanco fue pintado como el cuarto topónimo, se registró con el corte lateral de una muralla. E. Boban, afirma que esta localidad fue representada por dos muros cortados dispuestos en gradas y que Tenanco quiere decir "en el recinto o intramuros"20; R. Siméon, menciona que este es un nombre dado a gran número de localidades situadas en los departamentos de México, Puebla, Oaxaca, Chiapas, entre otros ${ }^{21}$; dentro de la obra de D. Chimalpáhin, se menciona la ciudad de Tenanco con el nombre de Tzacualtitlan Tenanco, fundada por el chichimeca y tlatohuani Cuahuitzatzin, poniéndole como tercer nombre Chiconcóhuac, aunque también se reconoce como la segunda cabecera de la ciudad de Amaquemecan Chalco ${ }^{22}$; J. Corona menciona que Tetenanco era parte de los "22 pueblos que formaban la provincia de Tepeyacac [y que] estos pueblos se localizaban en el centro y sur del Estado de Puebla"23; reforzando el escenario geográfico, $\mathrm{CH}$. Gibson, sitúa a la provincia de los chalca al sudeste de la Cuenca de México y menciona a Tenango como una comunidad principal en los periodos prehispánico y colonial ${ }^{24}$.

La información que nos proporciona Siméon y el registro del topónimo de Tenanco en las láminas

20 Boban, 289.

21 Siméon, 474.

22 Chimalpáhin, 329.

23 Corona, 36.

24 Gibson, 18. 
16, 19 y 22 de la MATRÍCULA DE TRIBUTOS, no nos permite una ubicación física exacta de este lugar; en las tres láminas de la MATRÍCULA, aparece el glifo de Tetenanco, el cual pertenece, según J. Corona y M. Sepúlveda a los actuales estados de Guerrero y Puebla.

Desde nuestro particular punto de vista, ocurre lo mismo que con el topónimo de Tecpan; tenemos información que ubica a Tenanco en distintos puntos geográficos, pero con las citas de D. Chimalpáhin, CH. Gibson, J. Corona y M. Sepúlveda y con las cuales concordamos, podemos entonces ubicar al barrio de Tenanco dentro de los límites de la región chalca; esto a consideración del tipo de tributo que debía pagar la gente de Tenanco, el cual está pintado dentro de los códices 028 y 108 .

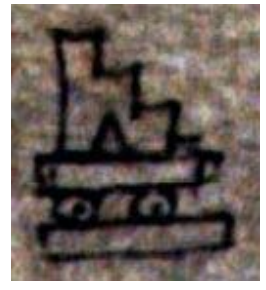

Imagen 5. Topónimo de Tenanco, Códice 028, BNF.

El quinto topónimo corresponde a la localidad de Quecholac, que está plasmado por el movimiento circular del agua, alrededor del glifo aparecen unas especies de caracoles que son representaciones de chalchihuites. E. Boban, nos dice que Quecholac significa "entre las aguas de quecholli’25; por otro lado R. Siméon, citando a Clavijero menciona que Quecholac es una población situada al oriente del estado de Tepeyacac, en la región de los popoloca ${ }^{26} ; \mathrm{H}$. Martínez, anota que el espacio territorial de Tepeaca comprende una pequeña porción de la región central-

25 Boban, 289.

26 Siméon, 420. 
sur del estado de Puebla y que a esta región corresponde el pueblo de Quechulac ${ }^{27}$; por otra parte, en la Relación de Tepeaca (1985: 227-228) se dice que esta provincia tiene tres pueblos cabecera, uno de ellos lleva el nombre de Quecholac, el cual es traducido como “agua donde está un pájaro parado que se nombra quechol"; también se da la traducción de este topónimo como "lugar del agua de quechulli" o "de la pluma preciosa"28.

El registro del topónimo de Quecholac, aparece contenido dentro de la lámina número 22 de la MATRÍCULA DE TRIBUTOS, aunque cabe señalar que el glifo es distinto, pues según J. Corona, el glifo es un adorno de plumas de quecholli dentro de una vasija con agua, el cual traduce como "en el río o lago de los quecholis"29; M. Sepúlveda, traduce este topónimo como "en el río de los quecholes" ${ }^{\prime 30}$; mientras que C. Macazaga, retoma el mismo glifo de la MATRÍCULA, mencionando que lo que reposa sobre el agua es una borla con plumas de águila o cuauhpilolli "colgajo de plumas de águila" y no de quechol. ${ }^{31}$ Finalmente todos los autores apuntan que el topónimo de Quecholac, pertenece al actual estado de Puebla.

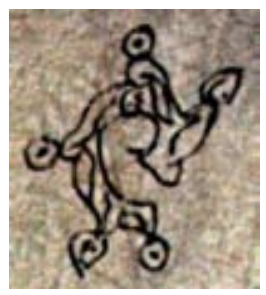

Imagen 6. Topónimo de Quecholac, Códice 028, BNF.

$27 \quad$ Hildeberto Martínez, Tepeaca en el siglo XVI, tenencia de la tierra y organización de un señorio (México: CIESAS, ediciones de la Casa Chata, $1^{\mathrm{a}}$ edición, 1984), 13.

28 Relaciones geográficas del siglo XVI: Tlaxcala, editor René Acuña (México: Instituto de Investigaciones Antropológicas, UNAM, 1985) 227,228.

29 Corona, 48-50.

30 Sepúlveda, 64.

31 César Macazaga Ordoño, Nombres geográficos de México, (México: editorial Innovación, 1978), 74-75. 
Ayocalco fue registrado como el sexto topónimo, fue representado a través de una especie de casa de estilo prehispánico pero con la textura de un caparazón de tortuga. E. Boban, lo traduce como "la casa de la tortuga" ${ }^{2}$. Este topónimo no ha podido ser localizado geográficamente aún.

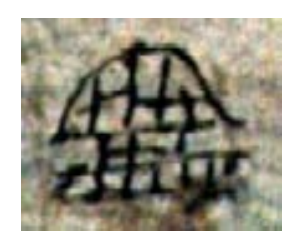

Imagen 7. Topónimo de Ayocalco, Códice 028, BNF.

San Nicolas es el séptimo topónimo, fue representado a través del glifo del agua, en la parte de abajo lleva la simbolización de los chalchihuites. Según E. Boban, se trata de un suburbio o barrio nuevo que fue fundado después de la llegada de los conquistadores ${ }^{33}$; dentro de las relaciones de Colhuacan, D. Chimalpáhin, afirma que los seis calpules chichimecas se trasladaron de Tizatépec a Cuitlatetelco, que ahora se llama San Nicolás, según el mismo D. Chimalpáhin, Cuitlatépec o Cuitlatetelco dista cuatro leguas y media de Chalchiuhmomozco que ahora se llama Amaquemecan ${ }^{34}$; de tal manera que podemos localizar a este barrio dentro de la zona chalca.

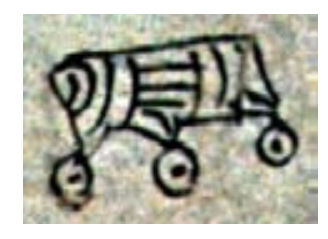

Imagen 8. Topónimo de San Nicolas, Códice 028, BNF.

32 Boban, 289.

33 Boban, 289.

34 Chimalpáhin, 139. 
El octavo topónimo es el de Atlaca, fue representado por medio de una red para pescar. E. Boban, citando a Molina, dice que son marineros ${ }^{35}$. Esta localidad no ha sido identificada geográficamente.

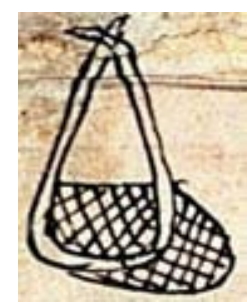

Imagen 9. Topónimo de Atlaca, Códice 108, BNF.

Según E. Boban, el noveno topónimo es el de Pochtecan o cerca de los Pochtecatl, nos dice que esta palabra significa mercader o negociante ${ }^{36}$; fue representado a través de un abanico ovalado que es el signo distintivo de los comerciantes. En la Relación de Tolnacuchtla se hace mención de un poblado llamado Hueypuchtla que se traduce como "grande feria"; R. Acuña, señala que Pochtlan significa "lugar de la Ceiba" y citando a B. Sahagún dice que era el nombre de un monasterio de los ministros de Yacatecuhtli, dios de los mercaderes; en la misma relación de Tolnacuchtla se dice que este pueblo y su comarca distan ocho leguas de la ciudad de México $^{37}$.

Entonces posiblemente por encontrarse dentro de los límites de la Cuenca pueda tratarse de la misma localidad que se representó dentro del códice 108 . Por su parte R. Siméon, nos dice que Pochtlan es una localidad situada al sur de Xochimilco ${ }^{38}$; C. Macazaga, señala que Hueipochtlan, 35 Boban, 289.

36 Boban, 290. 
deriva de huei "grande" y Pochtlan "cerca del patrono", del dios Yacatecuhtli, "cerca del gran Pochtlan" y también ubica este topónimo al sur de Xochimilco ${ }^{39}$.

Es importante resaltar que el topónimo de Hueypochtlan aparece registrado en la lámina número 9 de la MATRÍCULA DE TRIBUTOS, J. Corona, nos dice que los pueblos que aparecen en esta lámina estaban bajo el dominio tepaneca y que actualmente pertenecen a los estados de Hidalgo y México ${ }^{40}$. Hacemos notar que el glifo que fue pintado en la MATRÍCULA fue representado a través de la cabeza de un personaje masculino pintado de color rojo con una especie de pluma como tocado.

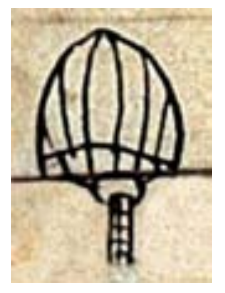

Imagen 10. Topónimo de Pochtecan, Códice 108, BNF.

El décimo de los topónimos es Petlachiuhque o Petlachiuh, que fue registrado por medio de un petate. Según E. Boban, el nombre de la localidad es Petlachiuh, de petlatl "estera de juncos" ", lo que indica que es un sitio donde se fabrican las esteras. Esta localidad no ha sido encontrada geográficamente.

37 Acuña, 141.

38 Siméon, 389.

39 Macazaga, 60.

40 Corona, 29.

41 Boban, 290. 


\section{O月 $9^{\circ}$ C ONGRESO \\ - i. CENTROAMERICANO \\ DE HISTORIA \\ Universidad de costa Rica}

Indexaciones: Repositorio de Revistas UCR, DIALNET, Latindex, REDALYC Directorio y recolector de recursos digitales del Ministerio de Cultura de España, Directory of Open Access Journals. Diálogos Revista Electrónica de Historia ISSN 1409-469X. Número especial 2008. Dirección web: http://historia.fcs.ucr.ac.cr/dialogos.htm

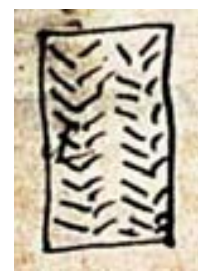

Imagen 11. Topónimo de Petlachiuhque o Petlachiuh, Códice 108, BNF. 